\title{
Erratum: Recent advances in analytical and bioanalysis applications of noble metal nanorods
}

\author{
Ilaria Mannelli • Maria-Pilar Marco
}

Received: 8 December 2010 /Accepted: 8 December 2010 /Published online: 22 December 2010

(C) Springer-Verlag 2010

\section{Erratum to: Anal Bioanal Chem DOI 10.1007/s00216-010-3937-8}

Regrettably, there are some mistakes in the reference numbers given in the text. The correct reference numbers are given below.

Page 2454:

Table 1: The correct reference number for Wang et al. 1999 is [37] not [36] and for Sau and Murphy 2004 it is [29] not [28].

Page 2463:

left column: [104] should be [84] (Varghese et al. 2008) right column: [105] should be [104] (Eghtedari et al. 2007). [106] should be [105] (Song et al. 2009). [107] should be [106] (Wang et al. 2005).
Page 2464:

left column: [108] should be [107] (Durr et al. 2007).

[107] should be [106] (Wang et al. 2005).

[109] should be [108] (Moskovits, 2005).

Page 2465:

left column: [110] should be [109] (Chaney et al. 2005). [111] should be [110] (Leverette et al. 2009).

[112] should be [111] (Shanmukh et al. 2006).

right column: [113] should be [112] (Chu et al. 2008).

[111] should be [110] (Leverette et al. 2009).

Page 2466:

left column: [114] should be [113] (Wang et al. 2010). [115] should be [114] (Lee et al. 2006). First [116] should be [115] (Yun et al. 2007). [114] should be [113] (Wang et al. 2010).

The online version of the original article can be found at http://dx.doi. org/10.1007/s00216-010-3937-8.

I. Mannelli $(\bowtie) \cdot$ M.-P. Marco

Applied Molecular Receptors Group (AMRg), IQAC-CSIC,

Networking Research Center on Bioengineering,

Biomaterials and Nanomedicine,

Jordi Girona, 18-26,

08034 Barcelona, Spain

e-mail: ilaria.mannelli@iqac.csic.es 